\title{
Analysis of the Characteristics of Sepsis After Chemotherapy for Lung Cancer
}

Geling Teng ( $\square$ tenggeling@163.com )

Shandong Provincial Chest Hospital

\section{CHEN Ying}

: Shandong Provincial Chest Hospital

\section{Zhao Wenwen}

: Shandong Provincial Chest Hospital

LI Xiangmei

: Shandong Provincial Chest Hospital

HU Hua

: Shandong Provincial Chest Hospital

\section{Case report}

Keywords: malignant tumor, hospital infection, septicemias, glucocorticoid

Posted Date: March 17th, 2021

DOI: https://doi.org/10.21203/rs.3.rs-305315/v1

License: (c) (i) This work is licensed under a Creative Commons Attribution 4.0 International License. Read Full License 


\section{Abstract}

\section{Background}

Nowadays, hospital infection have a high incidence of of sepsis in clinic for malignant tumor patients after chemotherapy, patients generally lack typical clinical manifestations in early stage, especially those patients with more complicated basic diseases, and their prognosis is poor.

\section{Case presentation}

In this report, we analyzed a lung cancer patients infected with enterobacter cloacae after chemotherapy, the clinical case data were collected, the course of its onset, diagnosis and treatment were analyzed, and the relevant literatures at home and abroad were reviewed for summary. The patient was elderly with underlying diseases, and was diagnosed with lung adenocarcinoma. He had a long history of glucocorticoid because of Interstitial pneumonia, and was given chemotherapy. The patient was complicated with sepsis after chemotherapy. Despite active treatment, the patient died of septic shock. There are many reasons for septic shock in patients.

\section{Conclusions}

The mortality of patients with malignant tumor complicated with nosocomial infection is high, which is closely related to the underlying disease and the application of glucocorticoid.

\section{Background}

Nowadays, hospital infection have a high incidence of sepsis in clinic, usually in the early stages the patient has no obvious discomfort, sudden onset is a typical clinical characteristic[1]. Pathogenic bacteria enter the blood circulation through the body weak barrier within a large number of rapid reproduction, at the same time release toxins, which cause the body of a series of pathology, even septic shock. The immune function of patients with malignant tumor is reduced. During the treatment, patients may receive surgery, repeated radiotherapy, chemotherapy and related immunotherapy, etc., which further reduces the immune function of patients and easily causes infection[2-4]. In this report, we analyzed the case of lung cancer after chemotherapy in patients with infectious shock, the final death cases complicated with sepsis. Through the analysis of the clinical characteristics and treatment process of patients, the risk factors of sepsis were further understood to improve the diagnosis and treatment ability of clinicians, and reduce the clinical morbidity and mortality.

\section{Case Presentation}

The 70-year-old male patient was admitted to the hospital on January 28, 2020 due to "lung lesions found in physical examination for one year", Physical examination: vital signs were stable, rales were heard in both lower lungs, and other signs were normal. Chest CT showed interstitial pneumonia and 
pulmonary infection (Figure 1). blood routine, C-reactive protein and calcitonin were normal. The sputum culture showed pseudomonas aeruginosa. According to the drug sensitivity test, the patient was given levofloxacin combined with piperacillin and tazobactam for anti-infection for 2 weeks, and the sputum culture of the patient recovered negative. In order to understand the changes of the disease, chest CT was rechecked (Figure 2), and the left lung disease was not improved. After communication with the patient, in order to further clarify the cause, CT-guided lung puncture was performed, and the pathological diagnosis was lung adenocarcinoma (Figure 3). The general condition of the patient was evaluated, and the PS score of the patient was 1 . After multidisciplinary consultation, chemotherapy was recommended, and pemetrexed combined with nedaplatin was given. The chemotherapy was successful and the patient had no obvious discomfort during the chemotherapy. However, on the second day after chemotherapy, the patient developed fever, accompanied by chills and diarrhea. Blood routine showed that the white blood cell count was significantly increased, C-reactive protein and procalcitonin were significantly higher than normal. Blood culture showed enterobacter cloacae, according to the drug sensitivity test, meropenem combined with polymyxin was given. After 10 days of anti-infection treatment, the body temperature gradually dropped to normal and the blood culture returned negative. However, the diarrhea did not improve. Routine stool and occult blood test showed no abnormalities. After consultation with gastroenterologists, the patient was given treatment such as improving flora imbalance and antidiarrhoea, but the stool frequency was still large and the improvement was poor. The patient's condition worsened sharply, including respiratory failure and shock. After multi-disciplinary consultation and rescue, anti-shock and fluid rehydration treatment were given, the patient's condition continued to deteriorate, shock was difficult to correct, and the patient died.

\section{Discussion And Conclusions}

Septicemic shock caused by nosocomial infection has a high clinical incidence at present [5], and its early symptoms are generally not obvious. It is a systemic infectious disease, mainly pathogenic bacteria invade human blood circulation, grow and multiply in large number, and the toxin produced infringes human immune defense function. The patient's condition is dangerous, the disease progresses quickly, and eventually leads to multiple organ failure and death. Some patients with chronic diseases with low immune function are bedridden for a long time, which makes the treatment more difficult and the prognosis is poor. The onset of early sepsis is insidious and the symptoms are atypical, especially when the systemic condition is poor, the patient only presents low fever, which is easy to be neglected clinically and even affects the diagnosis [6-7].

There are many reasons for nosocomial infection leading to sepsis, including serious basic diseases $\rrbracket$ long-term application of large amounts of immunosuppressant, radiotherapy can seriously damage the hematopoietic function of bone marrow and gastrointestinal function, leading to malnutrition and low immune function. Chemotherapy destroys the integrity of intestinal mucosa and causes bacterial invasion [8-10]. In this report, the patient was older, with a basic disease of hypertension, and had been lying in bed for 2 years since the operation of renal malignant tumor. In addition, the patient suffered from interstitial pneumonia and had been taking oral glucocorticoids. As an immunosuppressant, 
glucocorticoids would inhibit the immune function of the body after long-term use, laying a foundation for the occurrence of sepsis.

All the doctors in our department discussed this case. Before chemotherapy, the patient was free to move, had strong physical endurance and had a PS score of 0 . The patient had had lung puncture biopsy under electronic tracheoscope and guided by $\mathrm{CT}$, for these reasons, I consulted relevant literature on whether these two factors would cause pathogenic bacteria to enter the blood. Through a review of domestic and foreign literature, there is no relevant report so far, and it is almost impossible for pathogenic bacteria to enter the blood caused by lung puncture injury to cause septicemia. Another related factor was that the patient had retained an intravenous indwelling needle during normal intravenous infusion. The literature on this aspect was extensively read and analyzed.

The clinical application of venous indwelling needle can keep the venous infusion unobstructed, which is convenient for emergency treatment, reduces the workload of nurses and improves the nursing work efficiency; at the same time, it also reduces the pain of repeated venipuncture of patients, but it increases also the chance of infection [11-12]. Through literature analysis, we found that venous indwelling can lead to sepsis, but studies have shown that most of the pathogens of indwelling needle infection are common bacteria on the surface of the human body, such as staphylococcus epidermidis, corynebacterium diphtheriae, etc., while this patient was infected with enterobacter cloacae, so venous indwelling leading to sepsis is quite reluctant for our patient [13].

To sum up, the cause of sepsis in this patient is not related to the use of lung puncture and intravenous indwelling needle. The preliminary analysis is still closely related to the basic disease, long-term use of hormone, basic conditions of malignant tumor, and chemotherapy.

Enterobacter cloacae, belonging to Enterobacteriaceae, is widely distributed in human respiratory tract, urinary tract, soil, dairy products, vegetables and polluted water. Generally, it is not pathogenic or opportunistic pathogen, and rarely causes primary infection. When the body's immune function is low, it can lead to sepsis, meningitis and other serious infections [14]. It is a common opportunistic pathogen in clinic, and has become a common bacteria in hospital infection in recent years [15].Low immune function has become an important pathogenic factor of the bacteria; it is easy to lead to septicemia, rapid progress of the disease, diversified clinical manifestations, often involving multiple organ systems, which can leave serious sequelae, and even lead to septic shock and death.

In this case report, the patient developed high fever after chemotherapy, and the patient's blood routine showed bone marrow suppression, which is closely related to chemotherapy. However, the patient's bone marrow suppression occurred early, and appeared on the first day after chemotherapy. This is very different from the clinical experience that bone marrow suppression often appeared one week after chemotherapy, which may be related to the patient's low immune function and hypersensitivity to chemotherapeutic drugs.After the patient developed fever, we immediately took blood culture and gave active anti infection treatment according to our experience. Three days later, the patient's blood culture showed Enterobacter cloacae, which was extensively resistant. According to the drug sensitivity test, we 
adjusted the combination of antibiotics polymyxin and meropenem, and gave various measures to improve platelet, leukocyte and human immunoglobulin. Although active treatment was given, the patient's condition still deteriorated rapidly, leading to sepsis, and eventually developed into septic shock. Later, through fluid resuscitation, endotracheal intubation and other rescue measures, the patient still died.

In the course of treatment, our measures are correct. The failure of rescue is closely related to the severity of sepsis itself, the primary disease and basic conditions of the patient. In addition, the underlying diseases interstitial pneumonia and malignant tumor are independent risk factors for septic shock death.

The purpose of this case report is to analyze whether there is any regret in the diagnosis and treatment of the patient in my charge, and analyze the causes of the patient's morbidity and death, so as to provide other clinical doctors with ideas for clinical diagnosis and treatment of septic shock.

\section{Declarations}

\section{Acknowledgements}

Many thanks to the medical record room of our hospital for all the information provided on this case.

\section{Authors' contributions}

All authors contributed to the study conception and design. Patient management, data collection, and analysis were performed by all authors listed as physicians. Further data analysis was performed by Teng Geling and Hu Hua. Materials were provided by Chen Ying, Zhao Wenwen, and Li Xiangmei. The frst draft of the manuscript was written by Teng Geling, and all authors commented on previous versions of the manuscript. All authors read and approved the fnal manuscript.

\section{Funding}

This article was not funded.

\section{Availability of data and materials}

All data generated or analyzed during this study are included in this published article.

\section{Ethics approval and consent to participate}

Approval was obtained from ethics Committee of Shandong Chest Hospital

The procedures used in this study adhere to the tenets of the Declaration of Helsinki. Written informed consent was obtained from the the patient's son. Consent for publication Additional informed consent was obtained from the patient's son. 


\section{Consent for publication}

Additional informed consent was obtained from the patient's son.

\section{References}

[1] Guo J L, Huang L M, Xie Y C, et al. Analysis of drug sensitivity and risk factors of multidrug-resistant bacteria in patients with malignant tumor [J]. Chinese Journal of nosocomial infection, 2017, 27 (10): 2194-2197.

[2] Xu QW, Zhang Q, Hu Ming, et al. Investigation of infection in patients with malignant tumor and analysis of related factors [J]. Chinese Journal of nosocomial infection, 2016, 26 (11): 2526-2528.

[3] Jiang Z C, Zhu S J, Dong JD, et al. Analysis of influencing factors of nosocomial infection in patients with malignant tumor [J]. Chinese Journal of nosocomial infection, 2016, 26 (8): 1781-1783

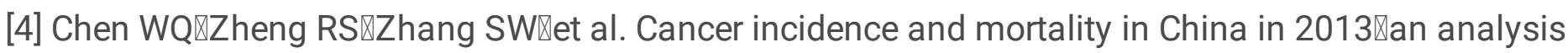
based on urbanization level『J囚. Chin J Cancer Res, 2017, 29ه18囚1-10.

[5] Li XX, Wang Y, Zhu L, et al. Clinical characteristics, pathogen distribution and drug resistance of 55 cases of neonatal sepsis with carbapenem resistant gram negative bacteria [J]. Chinese Journal of nosocomial infection, 2019,29 (16): 2500-2504.

[6] Han JL, Zhang XLi, Wang YF, et al. Preterm and full-term newborns comparative analysis of clinical characteristics of sepsis [J]. China maternal and child health, 2016, 31(6):1199-1201.

[7] Chen T, Ma HJ, Yang J, et al. Related factors and pathogens of neonatal sepsis [ $\mathrm{J}$ ]. Chinese Journal of preventive medicine, 2018, 52(10): 1023-1028.

[8] Zhang JL, Ni MM, Ji YH, et al. Cancer patients hospital of cancer hospital Investigation and analysis of infection. Chinese Journal of nosocomial infection, 2011, 21 (7): 1334-1336.

[9] Zhang Y,Wang Q,Yin Y,et al.Epidemiology of carbapenemresistant Enterobacteriaceae infections:report from the China CRE Network.Antimicrob Agents Chemother,2018,62(2):e01882-17.

[10] Banerjee R,Humphries R.Clinical and laboratory considerations for the rapid detection of carbapenem-resistant Enterobacteriaceae. Virulence,2017,8(4):427-439.

[11] Chen MJ. Nursing progress of fixation methods of intravenous indwelling needle in children in China [J]. Contemporary nurses, 2017 (4): 6-8

[12] Yang HWi, Zhang NW. Logistic regression analysis on risk factors of indwelling needle related phlebitis after general surgery [J]. Chinese Journal of nosocomial infection, 2011, 21 (22): 4709-4711 
[13] Shu Y, Mao SF, Yu XL, et al. Study on the correlation between the timing of PICC catheterization and phlebitis in patients with hematological diseases [J]. Journal of nursing education, 2012, 27 (11): 10061007区

[14] Lu Deyuan. Medical microbiology. 3rd Edition. Beijing: People's Health Publishing House, 1989, 139140.

[15] Zhou G, Shen DX, Luo YP, et al. Distribution of clinical Enterobacter cloacae infection. Chinese Journal of nosocomial infection, 2006, 16 (3): 340.

\section{Figures}

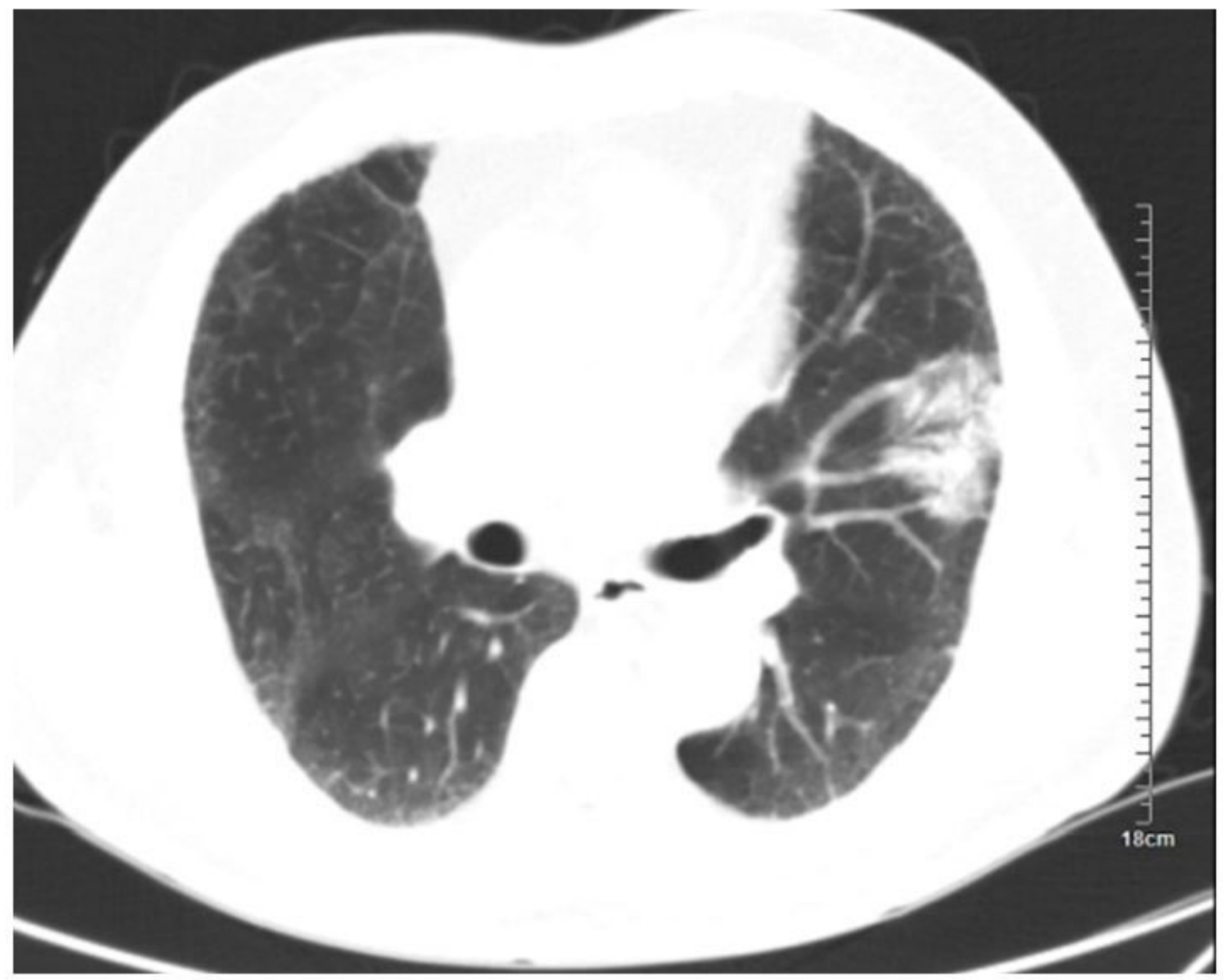

Figure 1 
patchy high-density lesion in the lingual segment of the upper lobe of the left lung, partly with GGO

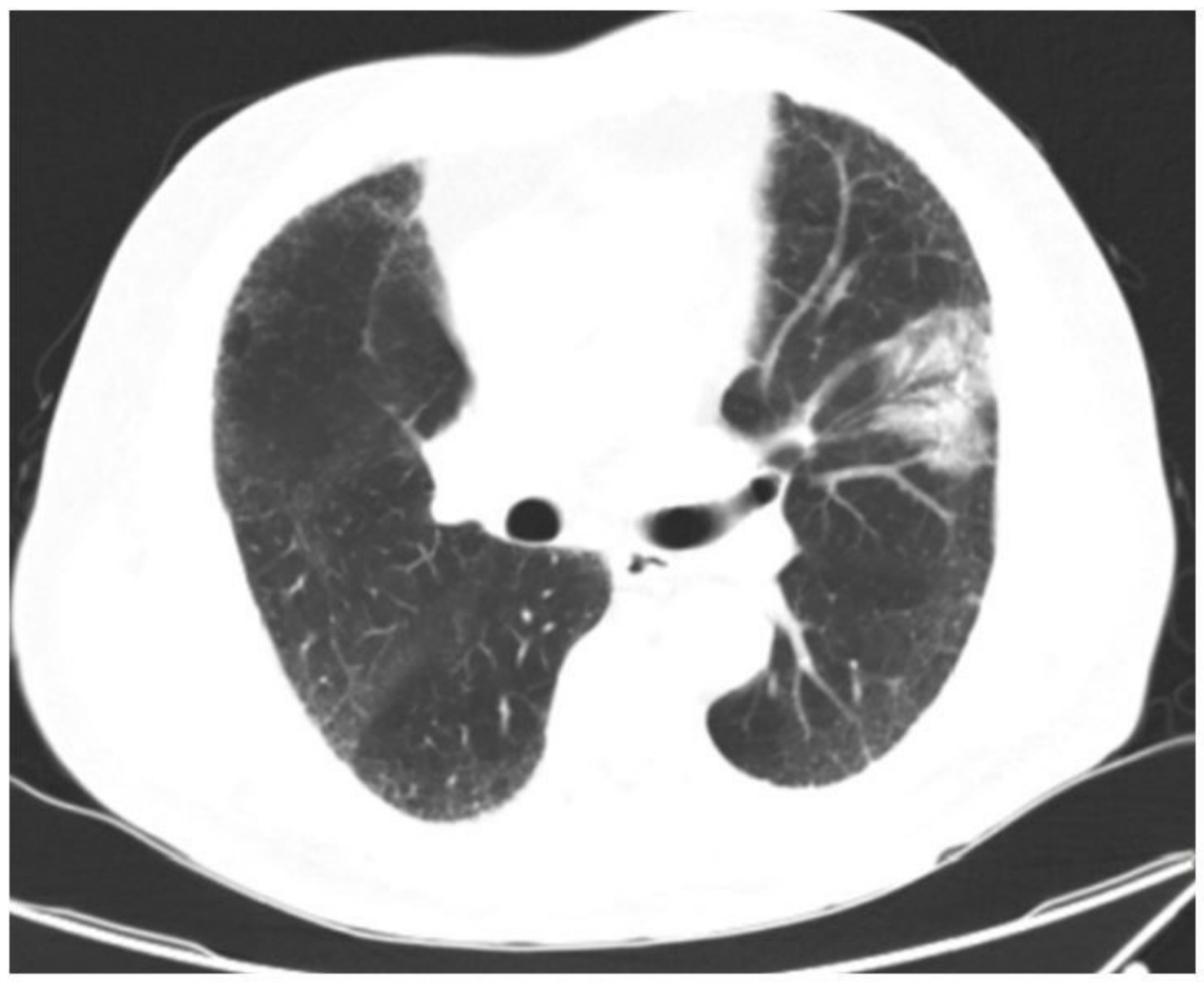

Figure 2

There was no improvement of pulmonary lesions and no absorption after anti-infection treatment 

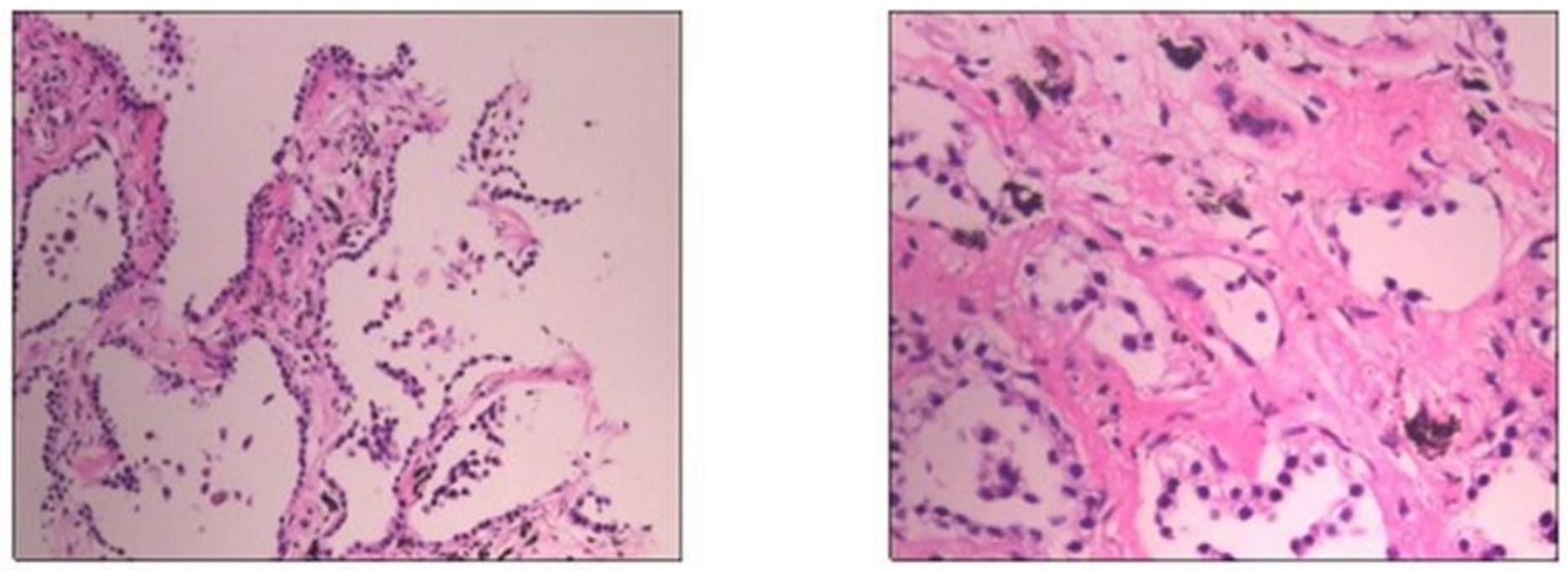

\section{Figure 3}

Lung biopsy revealed adenocarcinoma (adherent type and acinar type). 